\section{Not empirical, but observational or experimental}

\section{SIR}

The only "unfortunate" aspect of empirical work in medical ethics is the word "empirical", and its use in an editorial in the fournal of Medical Ethics.' Of all medical practitioners (used in the widest sense), philosophers and ethicists should be the most careful in their use of words, because their work largely consists in words.

Empirical has one of two conflicting meanings, ${ }^{2}$ the intended one discernible only by context. In the editorial, empirical meant "experimental", but the other meaning was intended in another recent editorial": "The recommendation ... is not based on any evidence but is purely empirical".

Anything that makes ethics more relevant to everyday clinical medicine is welcome. For that reason "researchbased", or "experimental", or "observational" medical ethics are all worthwhile.

\section{References}

1 Hope T. Empirical medical ethics. fournal of Medical Ethics 1999;25:21920.

2 Brewin TB. Empirical: one word, two meanings. Fournal of the Royal College of Physicians of London 1994;28:78-9.

3 Hughes G. Fitzharris P. Managing acute anaphylaxis. British Medical fournal 1999;319:1-2.

NEVILLE GOODMAN

Consultant Anaesthetist

Southmead Hospital

Bristol BS10 5NB

\section{(In)valid consent of advance directives}

SIR

Campbell's article ${ }^{1}$ raises the interesting suggestion that people suffering severe pain may not be able to make a voluntary choice for euthanasia. Per- haps his concept might equally apply to those "in extremis" from other stresses.

Several of us may have experienced "near death" situations, but it is arguable whether these are necessarily the same as the actual final moments we all shall face.

Some may face death with composure and (relative?) equanimity - but many are plagued by doubts and anxieties. Such doubts and anxieties may well be as challenging as the severe pain that our culture concentrates on. In which case, Campbell's thesis argues against euthanasia in general.

Flew's riposte ${ }^{2}$ seems to miss Campbell's comments about the time lapse between making an advanced directive and its implementation. Difficulties can and do occur in clinical practice. Flew states: "What the doctors concerned surely wanted to know .... is what those patients actually would have wanted had they been in a position to consider the issues calmly and to express themselves without the distraction caused by such extremes of pain. Clearly patients who are in conditions of the kind described by Dr Campbell cannot do this at the time of their afflictions. But they could and should previously, when they were fit and well, have signed an advance directive...."

This may work in an idealised, theoretical world - but is fraught with difficulty, if not danger, in the world of real doctors - and patients whose lives are at stake. For example, I had a lady in her mid-eighties with severe pneumonia a few years ago, who was hospitalised with severe dehydration and moderate delirium. According to the referral letter from her family doctor her past medical history included only mild arthritis and slight benign senescent forgetfulness. Her daughter, who came with her, insisted that nothing active should be done for mother: they had repeatedly discussed the possibility of mother developing a terminal illness and decided that mother wanted to die in peace.

Following gentle discussion with the daughter we gradually arrived at a mutually acceptable plan:

1. Mother should be nursed in hospital, in bed and in a quiet, single room to provide her with a comfortable, calm, dignified environment.

2. Mother should be given paracetamol to alleviate any pain/distress/ discomfort from the infection and pyrexia.

3. Mother should be given parenteral fluids subcutaneously (hypodermoclysis) to alleviate the dehydration.

4. Mother should be relieved of her hypoxia and given oxygen (by nasal specula).

5. The daughter would not agree to mother being given antibiotics, even though to the medical staff it seemed likely that the current illness could be cured without residual disability. The daughter agreed, however, to nurses putting antibiotics onto the bed-table in front of mother for her to take or leave. Each delivery was accompanied by a clear explanation of what was being left and why but no pressure was put on mother to take the tablets. The decision was left to her.

Mother duly recovered. On the morning of leaving hospital mother exclaimed in a loud voice in front of daughter, other ward patients, visitors and collected staff that she resented the doctor "getting her better". Fortunately I had the presence of mind to respond: "I didn't get you better. You elected to take the antibiotics which were only put in front of you. No pressure was put on you to take them!" 